\title{
Removal torque and bone adherence to dental implants surface
}

\begin{abstract}
Dental implant surface treatment may enhance the process of osseointegration. The aim of this study was to analyze the influence of two surface treatments on insertion and removal torque and the bone adherence to its surface after removal. Dental implants with acid surface treatment (Porous $=\mathrm{P}$ ) and fluoride surface treatment (Porous Nano=PN) were inserted in rabbit tibia with different torques. Implants were removed after osseointegration at 40 days of implantation. The insertion (IT) and removal (RT) torques were measured using a manual digital torquemeter. Removed dental implants were further analyzed by SEM to measure amounts of adhered bone to the implants surface. Significant differences between insertion and removal torques and between two types of implants were analyzed by Wilcoxon and Mann-Whitney tests, respectively. The IT of dental implants ranged from $20-40 \mathrm{~N} . \mathrm{cm}$, and the mean value was $30 \mathrm{~N} . \mathrm{cm}( \pm 8.16)$. The mean RT for both implants on day 0 was $33.33 \mathrm{~N} . \mathrm{cm}( \pm 11.5)$. On day 40 th, that mean was $28.40 \mathrm{~N} . \mathrm{cm}( \pm 3.67)$ and 30.15 N.cm $( \pm 1.34)$ for P and PN ( $>0.05)$, respectively. The percentage of surface with bone adhered was $26.55 \%( \pm 5.29)$ for $\mathrm{P}$ and $35.15 \%( \pm 6.92)$ for $\mathrm{PN}(\mathrm{p}>0.05)$. Porous implant may be more prone to present bone adhered to its surface than Porous Nano implant.
\end{abstract}

Keywords: dental implants, torque, osseointegration
Volume 8 Issue 2 - 2017

Carina M Silva Boghossian,' Luciane Ferreira Negrao,' Celso Renato de Souza Resende,' Carlos Nelson Elias, ${ }^{2}$ M rcio Eduardo Vieira Falabella, ${ }^{3}$ Denise Gomes da Silva'

'Department of Periodontics, University of Grande Rio, Brazil ${ }^{2}$ Department of Material Science, Military Institute of Engineering (IME), Brazil

${ }^{3}$ Federal University of Juiz de Fora, Brazil

Correspondence: Carina M Silva-Boghossian, Department of Periodontics, University of Grande Rio, University of Grande Rio, I I60, Odontologia, Duque de Caxias, Rio de Janeiro, Brazil, Email carinabogho@unigranrio.edu.br

Received: September 14, 2017 | Published: September 22 2017
Abbreviations: $\mathrm{P}$, porous; $\mathrm{PN}$, porous nano; IT, insertion torque; RT, removal torque; SEM, scanning electronic microscopy

\section{Introduction}

The primary stability is an important factor in achieving a predictable dental implant treatment. Therefore, in order to reach longitudinal success, it is necessary to achieve and to maintain the primary stability as basic pre-requirement. ${ }^{1,2}$ The primary stability can be considered the first stabilization that is obtained immediately after implant insertion. ${ }^{3}$ Thus, the insertion torque during implant installation may be an indicative of implant survival. ${ }^{4}$ However, the initial stability may not be a factor that assures the osseiointegration, because it can be influenced by bone properties, surgical techniques and implant design. ${ }^{3}$ Consequently, the measurement of the insertion torque could not be the only parameter to predict the osseointegration success rate. Thus, there are studies that evaluate the implants surface treatment to investigate what may influence the prognosis..$^{5-7}$ After the initial wound healing, some features determine the secondary stability as the biological response to the surgical trauma, the conditions of the wound healing and the material of the implant perse. ${ }^{8}$ Therefore, there is an increase in the contact in the bone-implant interface. ${ }^{8}$ At the same time, the presence of osteocytes in the bone remodeling in the boneimplant interface as well as the amount of sclerostin, osteoprotegerin and RANKL influence this remodelling. ${ }^{9}$ Thus, when a traumatic surgery is performed or load is applied before the right time can lead to a tissue injury, resulting in bone resorption. ${ }^{9}$ The design of the dental implant has evolved to improve its mechanical, functional and esthetic efficiency. ${ }^{3-8}$ Nonetheless, the events that occur in the bone-implant interface are still in need of further investigation. ${ }^{6,7}$ Having that in mind, studies involving implants with different surface treatments and the understanding the effects of different torques are important to a more comprehensive knowledge of this surfaces and the interaction with the bone tissue. ${ }^{1-8}$ Thus, the aim of this study was to analyze the influence of the insertion torque applied to titanium dental implants with different surface treatment (acid and fluoride) on the bone adherence to the implant surface after removal from rabbit tibia by scanning electronic microscopy (SEM) after 40 days of implantation.

\section{Methods}

Six young male New Zealand rabbits weighting about $3 \mathrm{~kg}$ and in good health were selected for the study. The study protocol was approved by the Evaluation Committee on the Use of Animals in Research of the Federal University of Rio de Janeiro, Brazil (\#ODONTO014). Two types of commercially pure titanium dental implants, P (Conexão Sistema de Protese Ltda, São Paulo, SP and Brazil) and PN (Conexão Sistema de Protese Ltda), measuring $3.5 \mathrm{~mm} \times 5 \mathrm{~mm}$ were placed in the left tibia of each rabbit. P implant presented a surface treated with acid etching $\left(\mathrm{H}_{2} \mathrm{SO}_{4}\right.$ and $\left.\mathrm{HCl}\right)$; while PN implant surface was treated as in P surface plus fluoride solution. Prior to surgery, the animals were weighted and anesthetized with an intramuscular combination of ketamine $1 \mathrm{~g}$ (Dopalen, $0.2 \mathrm{mg} /$ $\mathrm{kg}$, Sespo Ind. \& Com. Ltda, Paulina, SP, and Brazil) and xylazine $2 \%$ (Anasedan, $0.1 \mathrm{ml} / \mathrm{kg}$, Sespo Ind. \& Com. Ltda). After that, 
trichotomy of the left tibia was done, and antisepsis of the local was performed with iodized alcohol $10 \%$. Then, an incision was made to expose the bone of the tibia. Under constant irrigation with sodium chloride $0.9 \%$, the bone was prepared for the installation of the implants. The insertion torque was recorded with a manual digital torquemeter (Digital Torque Wrench, Torque Meter Lutron TQ-8800, Taipei, Taiwan), which was registered in Newton centimeter (N.cm). Periosteal suturing was performed with absorbable suture (vicryl 4-0, poliglactina 910, Ethicon), and epithelial suturing with nylon suture 4-0. Post-operatively, the animals received intramuscular injection of antibiotics (benzilpenicilina benzatina 1.200.000 U, $40.000 \mathrm{UI} / \mathrm{kg}$, Benzetacil, Eurofarma Laboratorios LTDA, São Paulo, Brazil) in one single dose, and subcutaneous anti-inflammatory (Flunixinmeglumine $1 \%$, injectable Banamine, Schering-Plough Animal Health, Rio de Janeiro, Brazil) every $24 \mathrm{~h}$ for 3 days. The same operator performed all procedures. At day 40th, animals were sacrificed using an intracardiac injection of potassium chloride $10 \%(10 \mathrm{mg} / \mathrm{kg})$. Following that, the implants were removed, while removal torque was measured as described above. Afterwards, removed implants were submitted to SEM. Topographical analysis was performed on removed implants using SEM. For that purpose, the samples were fixed in sample holders using conductor tape and, then, placed in the analysis chamber of the SEM (FEG/EDS-Philips XL 30 FEG) with Oxford Link x-ray detector EDS. Secondary electrons were used at $25 \mathrm{kV}$ acceleration rate.

The data were analyzed using a statistical package (IBM SPSS Statistics 19, IBM, Armonk, NY, EUA). The mean values and standard deviation of the insertion and removal torques were obtained. The measurement of the surface area covered by bone tissue adhered over the implant surfaces after their removal was performed using ImageJ software (http://imagej.nih.gov). Through Image J tools, the area covered by adhered bone was delimited and its measurement computed by the software. Then, a proportion of covered area with adhered bone in relation to the total implant surface was obtained for both types of implants. Significant differences between both types of implants were investigated by Mann-Whitney test, and between insertion and removal torques by Wilcoxon test. The level of significance was established at 5\%.

\section{Results}

The surgical procedures were uneventful with no indications of infection or inflammation during postoperative period. All implants were osseointegrated. Figure 1 show the insertion and removal torques applied to $\mathrm{P}$ and $\mathrm{PN}$ implants installed in the animals. The value for insertion torque ranged from 20 to $40 \mathrm{~N} . \mathrm{cm}$ and the mean value was $30 \mathrm{~N} . \mathrm{cm} \pm 8.16$ in both groups. The mean values of removal torque for P implants at day 40 was $28.40 \mathrm{~N} . \mathrm{cm} \pm 3.67$, and for PN implants, $30.15 \mathrm{~N} . \mathrm{cm} \pm 1.34$. There was no difference between applied torques at the observation times ( $>0.05$; Wilcoxon test). Surface morphology of the P and PN implants after removal are presented in Figure 2. The proportion of surface covered by adhered bone tissue was calculated and its mean ( \pm standard-deviation) is shown in Figure 3. This proportion showed a tendency to be higher for P implant (35.15 $\% \pm 6.92)$ than PN $(26.55 \% \pm 5.29)$. The statistical analysis shows no different proportion of surface covered by bone tissue among $\mathrm{P}$ and PN implants.

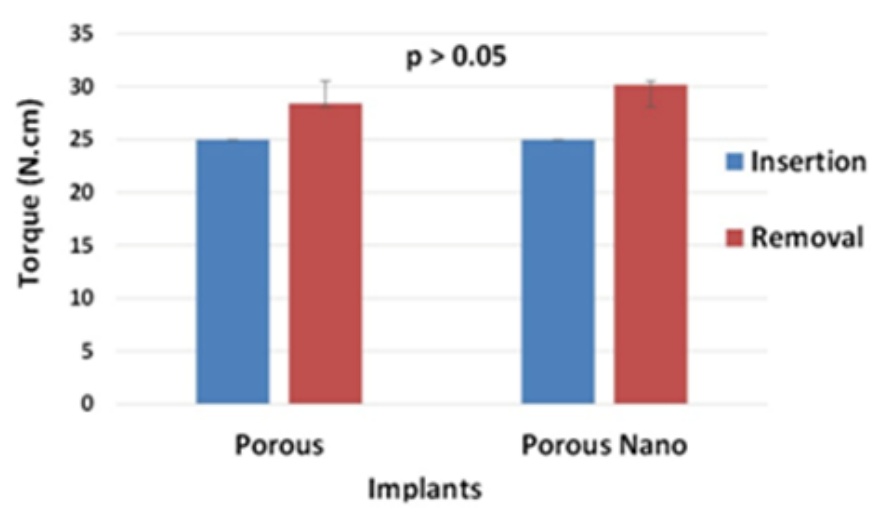

Figure I Insertion and removal torques applied to Porous and Porous Nano implants. $\mathrm{P}$ value $>0.05$, Mann-Whitney test (for comparison between types of implants) and Wilcoxon test (for comparison between insertion and removal torques).

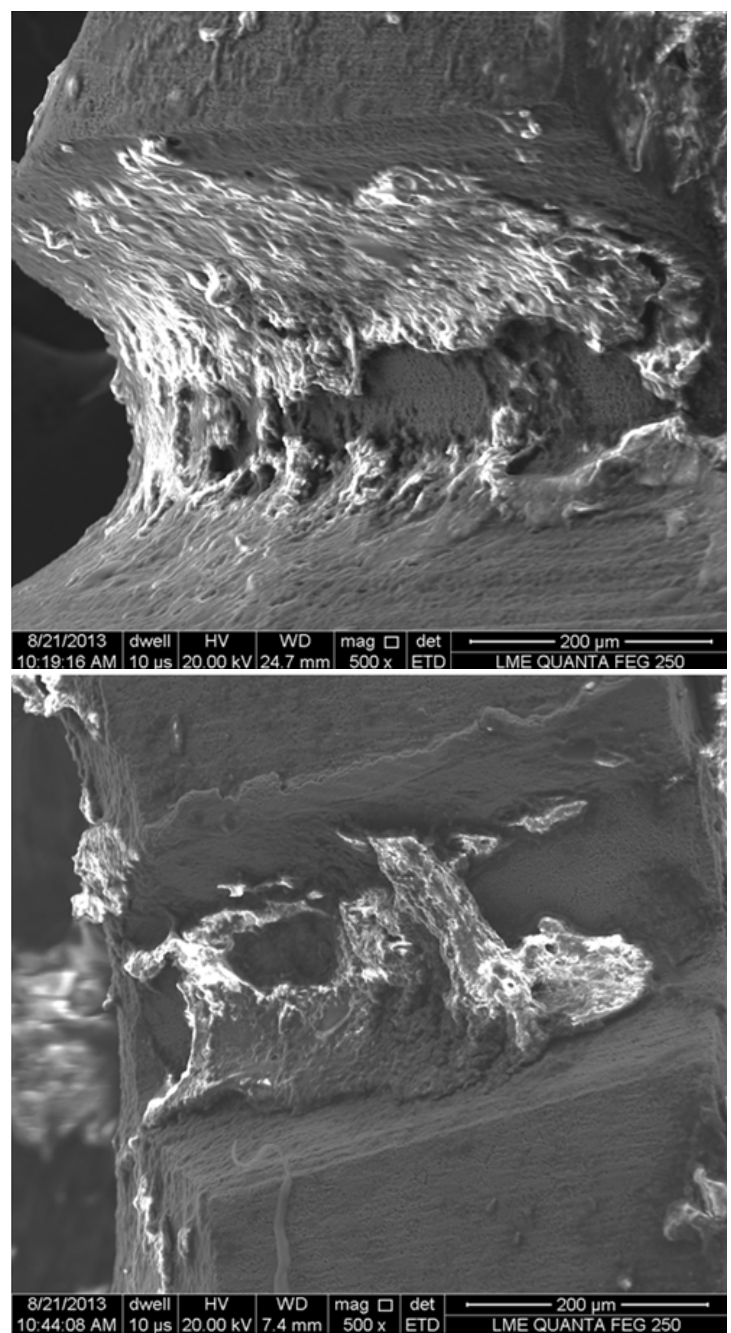

Figure 2 Scanning electron microscopy micrographs with 500x magnification of the removed implants after 40 days of installation. (A) Porous implant. (B) Porous Nano implant. 


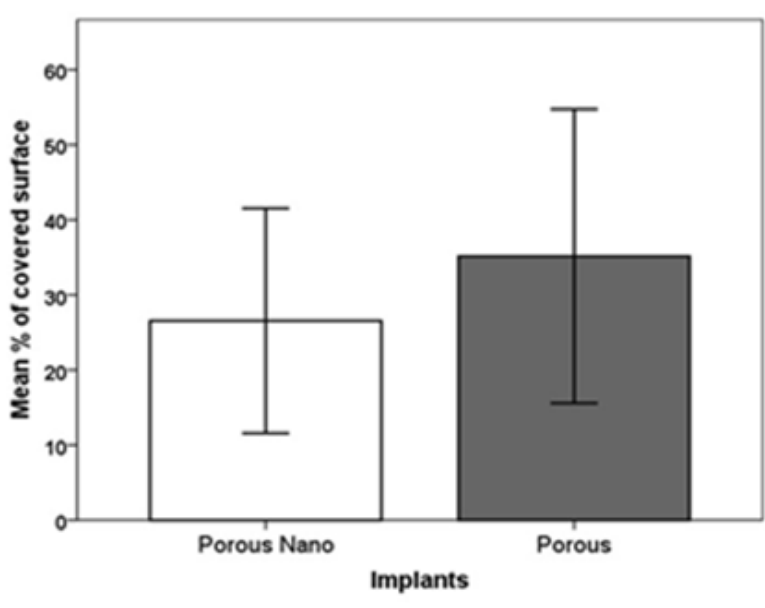

Figure 3 Mean percent of surface covered by bone tissue analyzed by scanning electron microscopy. Columns represent mean percent, and error bars represent \pm standard-deviation; $\mathrm{p}$ value $>0.05$, Mann-Whitney test.

\section{Discussion}

The aim of current study was to evaluate the insertion and removal torques of dental implants with two different surface treatments in rabbit tibia, and the bone adherence on the surface of both types of implants. In order to answer this objective, the study used an animal model that is largely used in this type of study design. ${ }^{1-12}$ Furthermore, the observation time of 40 days is also comparable to other studies. ${ }^{1-12}$ It has been shown that, in this period, it is possible to evaluate the initial events that occur in the bone-implant interface, which are important for the osseointegration success. ${ }^{8}$ Nonetheless, it should be remarked that findings from studies involving animals may not be directly applied to clinical situations. Conversely, clinical studies are usually limited to clinical and radiographic parameters or, in some cases, to extraction of failing implants. ${ }^{13-15}$ Thus, the use of experimental animals is justified in studies of biological principles. Particularly in rabbits, the bone tissue of the tibia may be comparable to the human alveolar bone tissue, which can allow comparisons to similar events in osseointegration. ${ }^{1-12}$ Regarding animals sample size, a variety of number of animals can be found in the literature ${ }^{1-17}$ in Cho \& Park, ${ }^{16}$ for instance, 7 rabbits were studied, and in Dasmah et al. ${ }^{6} 8$ rabbits were studied. In the current study, similar number of animals comparable to those studies was employed. Calculations with open access software WINPEPI (PEPI for Windows) using current standard-deviation values found in both groups $( \pm 6.92 ; \pm 5.29)$, it was found that for a difference of $10 \%$ between groups 6 animals would be sufficient. For ethical reasons, it is noteworthy to remember that an excessive number of experimental animals are always questionable. ${ }^{18}$ Regarding osseointegration stability, some factors should be considered, such as the biocompatibility of the material, the implant design, the implant surface, the surgical protocol, and the time after surgery. ${ }^{1-19}$ Moreover, acid-etched implants may present enhanced bony anchorage when compared to machined implants. ${ }^{1,2}$ In the current investigation, it was demonstrated that at 40th days the bone tissue was already adhered to the installed implants as seen by SEM analysis (Figure 2). Although there was no significant difference between both implant types, $\mathrm{P}$ implant surface demonstrated higher bone tissue coverage compared to PN implant. That finding could be partially explained by the surface treatment of the implant rather than the applied insertion torque, which was similar for both implants. It was demonstrated by Mazzo et al. ${ }^{5}$ that the implant surface treatment increases their primary stability. According to the study of Marin et al. ${ }^{8}$ an increased as-turned surface microtopography may result in enhanced biologic responses and biomechanical fixation values. Despite the lack of significant differences among implants in the current study, it is possible that surface treatment, which induces nanometric features, changes the bone-implant interface response ${ }^{20}$ Other possible explanation for P implant to present a tendency to a better behavior could be due to mechanical interlocking between the surface and bone. ${ }^{8}$ On the other hand, a previous report has shown that fluoride implant treatment may show a higher degree of osseointegration and stability when compared to control implants with machined surface texture. ${ }^{5}$ Moreover, literature shows that implant surface can impact in the insertion torque. ${ }^{4-23}$ Nevertheless, the current findings have demonstrated that the insertion torque applied to P and PN implants were not significantly different. It has been described that when a low insertion torque value is achieved it may lead to an initial low amount of bone-implant contact formation compared with higher torque applied to the same type of implant 11. On the other hand, some authors have considered that a high insertion torque increases the concentration of compressive stress. ${ }^{22-24}$ It may lead to an overpressure applied to the implant, and, consequently, a continuous compression may be added to the surrounding bone, creating bone fractures and crack nucleation. However, factors as dimension of the roughness, the presence of contaminants and surface cleanness are important for the biological response in short and long term analysis. ${ }^{3}$ Nonetheless, it may be recommended to allow a maximum contact between bone and implant during its installation to reduce fibrous tissue in that interface. ${ }^{17}$ The insertion torques (mean of $25 \mathrm{~N} . \mathrm{cm}$ for both implants) produced are considered from low to medium. ${ }^{4-22}$ On the contrary, the mean removal torque in PN implants removal $(30 \mathrm{~N} . \mathrm{cm})$ is considered from medium to high (between 30 and $50 \mathrm{~N} . \mathrm{cm}$ ); while for P implants (mean of $28 \mathrm{~N} . \mathrm{cm}$ ) is considered from low to medium, according to the classification proposed by Walker et al. ${ }^{4}$ Conversely, a greater removal torque required to remove implants may be interpreted as an increase in the strength of bone integration. ${ }^{1}$ In the current investigation, the removal torque were always higher than the insertion torques for all installed implants, which is in agreement with a previous study. ${ }^{25}$ However, the current investigation has demonstrated that there are no significant differences between insertion and removal torque for both types of implants, Porous and Porous Nano. There is a trend in a higher bone adherence to the Porous implant surface when compare to the one of Porous Nano implant. The insertion torque seems to be always lower than the removal one, regardless of the implant surface.

\section{Conclusion}

Porous implant may be more prone to present bone adhered to its surface than Porous Nano implant.

\section{Funding}

None.

\section{Acknowledgments}

This study was supported in part by Foundation for Research Financial Support in the State of Rio de Janeiro (FAPERJ), Rio de Janeiro, Brazil. 


\section{Conflicts of interest}

The authors declare that they do not have any conflict of interest regarding the present study.

\section{References}

1. Klokkevold PR, Johnson P, Dadgostari S, et al. Early endosseous integration enhanced by dual acid etching of titanium: a torque removal study in the rabbit. Clin Oral Implants Res. 2001;12(4):350-357.

2. Tabassum A, Meijer GJ, Wolke JG, et al. Influence of surgical technique and surface roughness on the primary stability of an implant in artificial bone with different cortical thickness: a laboratory study. Clin Oral Implants Res. 2010;21(2):213-220.

3. Elias CN, Rocha FA, Nascimento AL, et al. Influence of implant shape, surface morphology, surgical technique and bone quality on the primary stability of dental implants. J Mech Behav Biomed Mater. 2012;16:169-180.

4. Walker LR, Morris GA, Novotny PJ. Implant insertional torque values predict outcomes. J Oral Maxillofac Surg. 2011;69(5):1344-1349.

5. Mazzo CR, Reis AC, Shimano AC, et al. In vitro analysis of the influence of surface treatment of dental implants on primary stability. Braz Oral Res. 2012;26(4):313-317.

6. Dasmah A, Kashani H, Thor A, et al. Integration of fluoridated implants in onlay autogenous bone grafts - an experimental study in the rabbit tibia. $J$ Craniomaxillofac Surg. 2014;42(6):796-800

7. Elkarargy A. Biological functionalization of dental implants with fibronectin: a scanning electron microscopic study. Int $J$ Health $\mathrm{Sci}$ (Qassim). 2014;8(1):57-66.

8. Marin C, Granato R, Suzuki M, et al. Removal torque and histomorphometric evaluation of bioceramic grit-blasted/acid-etched and dual acid-etched implant surfaces: an experimental study in dogs. $J$ Periodontol. 2008;79(10):1942-1949.

9. Consolaro A. Osteocytes: On the central role of these cells in osseous pathobiology. Dental Press Implantology. 2012;6(2):20-28.

10. Dasmah A, Thor A, Ekestubbe A, et al. Marginal bone-level alterations at implants installed in block versus particulate onlay bone grafts mixed with platelet-rich plasma in atrophic maxilla. a prospective 5-year followup study of 15 patients. Clin Implant Dent Relat Res. 2013;15(1):7-14.

11. Duyck J, Roesems R, Cardoso MV, et al. Effect of insertion torque on titanium implant osseointegration: an animal experimental study. Clin Oral Implants Res. 2015;26(2):191-196.
12. Gotfredsen K, Berglundh T, Lindhe J. Anchorage of titanium implants with different surface characteristics: an experimental study in rabbits Clin Implant Dent Relat Res. 2000;2(3):120-128.

13. Buhara O, Uyanik LO, Ayali A, et al. Active implant periapical lesions leading to implant failure: two case reports. J Oral Implantol. 2014;40(3):325-329.

14. Quirynen M, Vogels R, Alsaadi G, et al. Predisposing conditions for retrograde peri-implantitis, and treatment suggestions. Clin Oral Implants Res. 2005;16(5):599-608

15. Silva GC, Oliveira DR, Vieira TC, et al. Unusual presentation of active implant periapical lesions: a report of two cases. J Oral Sci. 2010;52(3):491-494

16. Cho SA, Park KT. The removal torque of titanium screw inserted in rabbit tibia treated by dual acid etching. Biomaterials. 2003;24(20):3611-3617.

17. De Vasconcellos LM, Oliveira FN, Leite Dde O, et al. Novel production method of porous surface Ti samples for biomedical application. J Mater Sci Mater Med. 2012;23(2):357-364.

18. Kilkenny C, Parsons N, Kadyszewski E, et al. Survey of the quality of experimental design, statistical analysis and reporting of research using animals. PLoS One. 2009;4(11):e7824.

19. Franchi M, Orsini E, Trire A, et al. Osteogenesis and morphology of the peri-implant bone facing dental implants. ScientificWorldJournal. 2004;4:1083-1095.

20. Mendes VC, Moineddin R, Davies JE. The effect of discrete calcium phosphate nanocrystals on bone-bonding to titanium surfaces. Biomaterials. 2007;28(32):4748-4755.

21. Akça K, Chang TL, Tekdemir I, et al. Biomechanical aspects of initial intraosseous stability and implant design: a quantitative micromorphometric analysis. Clin Oral Implants Res. 2006;17(4):465-472.

22. Degidi M, Daprile G, Piattelli A. Determination of primary stability: a comparison of the surgeon's perception and objective measurements. Int J Oral Maxillofac Implants. 2010;25(3):558-561.

23. Elias CN, Oshida Y, Lima JH, et al. Relationship between surface properties (roughness, wettability and morphology) of titanium and dental implant removal torque. J Mech Behav Biomed Mater. 2008;1(3):234-242.

24. Sotto-Maior BS, Rocha EP, De Almeida EO, et al. Influence of high insertion torque on implant placement: an anisotropic bone stress analysis. Braz Dent J. 2010;21(6):508-514

25. Ueda M, Matsuki M, Jacobsson M, Tjellström A (1991) Relationship between insertion torque and removal torque analyzed in fresh temporal bone. Int J Oral Maxillofac Implants. 6(4):442-447. 